\title{
DESCARTE RESIDENCIAL DE RESÍDUOS DE INSULINOTERAPIA NO MUNICÍPIO DE JUAZEIRINHO, PARAÍBA
}

\author{
DISPOSAL RESIDENTIAL WASTE OF INSULINOTHERAPY IN \\ JUAZEIRINHO CITY, PARAÍBA
}

Raissa Daniel Trajano dos Santos ${ }^{1}$

Luana Layse Câmara de Almeida ${ }^{2}$

Tássya Rebecka Neves Araújo ${ }^{3}$

Adriana Amorim de Farias Leal ${ }^{4}$

Rômulo Moreira dos Santos ${ }^{5}$

RESUMO: OBJETIVOS: Identificar o manejo e gerenciamento dos resíduos gerados por pacientes diabéticos usuários de insulina e análogos. MÉTODO: Estudo de prevalência, com abordagem quantitativa, realizado nos meses de junho a dezembro de 2019, no Município de Juazeirinho, Paraíba, junto aos pacientes diabéticos cadastrados na Farmácia Básica, sendo a coleta de dados iniciada após a aprovação por comitê de ética CESED-PB, recebendo a CAAE $n^{\circ}$ 11669519.0.0000.5175. RESULTADOS: Participaram 23 voluntários, destacando-se o predomínio do sexo masculino. A maior parte dos entrevistados foi diagnosticada com diabetes mellitus há mais de 10 anos, sendo o tipo 2 o mais prevalente. Sobre o armazenamento dos resíduos gerados, foi mais relatado o uso de garrafas PET, entretanto, muitos relataram apenas o uso de sacos plásticos comuns, realizando o descarte final junto aos demais resíduos residenciais para a coleta de lixo municipal. Uma prática problemática citada pelos participantes foi em relação ao reaproveitamento da seringa descartável, reutilizando-as mais de uma vez. CONCLUSÃO: A falta de orientação ao usuário de insulina, assim como a falta de legislação para resíduos de saúde produzidos em residências, são os principais problemas para o gerenciamos inadequado dos RSS. Faz-se necessário o investimento na educação em saúde para todos os profissionais da saúde, para que possam repassar orientações de autocuidado a pacientes com doenças crônicas.

\footnotetext{
${ }^{1}$ Graduanda do curso de Farmácia da Unifacisa.

${ }^{2}$ Graduanda do curso de Farmácia da Unifacisa.

${ }^{3}$ Graduanda do curso de Farmácia da Unifacisa.

${ }^{4}$ Estudante do Programa de Pós-graduação em Saúde Coletiva da UFRN.

5 Farmacêutico, Mestre em Saúde Pública a e Professor dos cursos de Farmácia e Nutrição da Unifacisa.
} 
Palavras chave: Resíduos de Serviços de Saúde. Insulinoterapia. Diabetes Mellitus.

SUMMARY :Goals: To identify the management and management of residues generated by diabetic insulin users and analogues. Method: Prevalence study with a quantitative approach, conducted from June to December 2019, in the city of Juazeirinho, Paraiba, with diabetic patients registered at the Basic Pharmacy, and data collection started after approval by the ethics committee CESED-PB, receiving CAAE No. 11669519.0.0000.5175. Results: 23 volunteers participated, highlighting the predominance of males. Most respondents were diagnosed with diabetes mellitus over 10 years ago, with type 2 being the most prevalent. Regarding the storage of waste generated, the use of PET bottles was more reported, however, many reported only the use of common plastic bags, making the final disposal with other residential waste for municipal waste collection. A problematic practice cited by participants was regarding the reuse of the disposable syringe, reusing them more than once. Conclusion: Lack of insulin user guidance, as well as lack of legislation for homeproduced health waste, are the main problems for inadequate management of SSR. It is necessary to invest in health education for all health professionals, so that they can pass self-care guidelines to patients with chronic diseases.

Keywords: Health Services Waste. Insulin Therapy. Diabetes Mellitus. 


\section{INTRODUÇÃO}

O diabetes mellitus é um problema de saúde para todos os países e independe do nível de desenvolvimento. Em 2017, 424,9 milhões de pessoas viviam com diabetes, estimando-se que em 2045 esse número suba para 628,6 milhões. $\mathrm{O}$ diabetes e suas complicações são as principais causas de mortalidade precoce em diversos países. Em 2015, 4 milhões de pessoas, entre 20 e 79 anos, vieram a óbito (SDB, 2019).

Diabetes mellitus é um distúrbio metabólico complexo, resultante da destruição das células produtoras de insulina, hormônio que regula a glicose, ou da combinação da resistência a insulina e deficiência relativa a insulina, é caracterizada pela hiperglicemia persistente. A classificação do DM é fundamentada na sua etiologia e pode ser classificado em diabetes mellitus tipo1, diabetes mellitus tipo 2 ou diabetes gestacional. Os diversos tipos de diabetes podem ter influência genética, ambiental e biológica (SBD, 2019).

Conforme a Sociedade Brasileira de Diabetes (2019), para o tratamento do Diabetes Mellitus o controle glicêmico pode ser feito através de mudanças no estilo de vida e hábitos alimentares além do uso de drogas antidiabéticas orais com diferentes mecanismos de ação e introdução da insulina exógena diariamente quando necessário. Sendo a insulinoterapia um tratamento que requer o máximo de cuidado do usuário diagnosticado com diabetes mellitus (FECHINE et al., 2015).

Resíduos de saúde podem ser gerados em unidades de saúde, bem como em residências por pacientes portadores de doenças crônicas que necessitam de tratamento diário, como é o caso de pacientes portadores de diabetes mellitus. $\mathrm{O}$ tratamento desses pacientes requer o uso diário ou não de insulina e verificação da glicemia capilar. Tais procedimentos são considerados invasivos, além de ser uma fonte geradora de resíduos compostos por seringas, agulhas, lancetas e fitas reagentes (SANTOS; SANTOS; PALERMO, 2019). 
Entre os resíduos gerados em domicílio por pacientes diabéticos estão as canetas, frascos de insulina, agulhas, seringas, lancetas algodão e fitas reagentes. Estima-se que em um ano sejam usadas no setor doméstico mais de 7,5 milhões de seringas, sendo a maioria utilizadas por diabéticos. Muitos insulinodependentes não recebem orientações sobre a forma segura do descarte do perfuro cortantes, fazendo com que esses resíduos sejam tratados como lixo comum (FECHINE et al., 2015).

Geradores de serviços de saúde são todos os serviços com atividades relacionadas a atenção à saúde humana ou animal, inclusive os serviços de assistência domiciliar. Os resíduos decorrentes de atividades exercidas nos serviços de saúde necessitam de processos diferenciados no seu gerenciamento para a disposição final adequada, tendo em vista suas características. A classificação dos Resíduos de Serviços de Saúde é dividida em grupos: A (potencialmente infectantes), B (químicos), C (radioativo), D (comum) e $E$ (perfurocortantes), os quais devem seguir as etapas de segregação, acondicionamento, identificação, transporte interno, armazenamento temporário, tratamento, armazenamento externo, coleta, transporte externo e disposição final (BRASIL, 2018).

Os RSS do grupo "A" são materiais que possuem a presença de agentes biológicos e que podem apresentar riscos de infecção, são materiais como cultura de estoque de microrganismos, resíduos de laboratórios, amostras que contenham sangue ou líquidos corpóreos. Resíduos do grupo "B" são os que contém produtos químicos e apresentam risco a saúde pública e ao meio ambiente é o caso dos produtos farmacêuticos, resíduos de saneantes, reagentes de laboratório além dos produtos tóxicos, corrosivos, inflamáveis e reativos. Resíduos do grupo "C" são os resíduos radioativos, a exemplo dos rejeitos radioativos de laboratórios de pesquisa e ensino da área de saúde, serviço de medicina nuclear e radioterapia. Resíduos do grupo "D" são os que não apresentam risco biológico, químico ou radiológico a saúde ou a meio ambiente, é o caso do lixo doméstico como papelão, papel, sobra de alimentos. Os resíduos do grupo "E" são os materiais perfurocortantes a exemplo de agulhas, ampolas de vidro, lancetas, bisturi, laminas de barbear, escalpes e vidro quebrados nos laboratórios (BRASIL, 2018).

Conforme a RDC n²22/2018 os RSS devem seguir as etapas de segregação, acondicionamento e identificação. Recomenda-se que o resíduo seja segregado 
após a geração, acondicionados em material resistente a ruptura, vazamento obedecendo os limites de peso e capacidade dos recipientes e identificados conforme a classificação do resíduo em local de fácil visualização. No caso de resíduos gerados pelos serviços de atenção domiciliar, devem ser acondicionados e recolhidos por pessoa treinada e encaminhados a destinação final adequada (BRASIL, 2018).

Considerando as diversas fontes geradoras, as residências de pacientes diabéticos são apontadas como um local de geração de resíduos, tendo em vista que os procedimentos realizados são semelhantes aos de uma instituição de saúde. Ainda, que a Anvisa e o Conama regulamentem o manejo e disposição dos RSS, não há legislação específica para o manejo de resíduos domiciliares (SANTOS; RUIZ, 2018). Pacientes insulinodependentes são geradores de resíduos dos grupos A, B e E. Logo, é recomendado que o descarte de resíduos domiciliar seja feito em coletores específicos, os quais raramente estão disponíveis nas Unidades de Saúde para futuro retorno e descarte adequado (SANTOS; SANTOS; PALERMO, 2019).

A geração e o manejo de RSS em domicílios necessitam de uma atenção especial dos profissionais atuantes nas Equipes de Saúde da Família (ESF), considerando o risco aos usuários, familiares, coletores de resíduos urbanos e ao meio ambiente. O diálogo entre profissionais e comunidade é primordial afim de desenvolver ações de autocuidado e de saúde (ANDRÉ; TAKAYANAGUI, 2015). As lancetas e agulhas, enquadrados no grupo $E$, nomeados de perfurocortantes são resíduos altamente contaminantes, devendo ser separados e descartado em um recipiente rígido conforme NBR 13853/97 da ABNT. O descarte inadequado desses resíduos é uma ameaça, podendo contaminar o meio ambiente e os profissionais que trabalham diretamente com o lixo, tendo em vista o contato com objetos que estiveram em contato com o sangue humano, podendo transmitir HIV e hepatites $\mathrm{B}$ e C (CAVALCANTI, 2016).

Garcia e Zanetti-Ramos (2004) citado por Santos e Ruiz (2018) enfatizam não apenas a transmissão de doenças infecciosas, mas também a preservação do meio ambiente, considerando que o risco é caracterizado pelo gerenciamento inadequado e contaminação do local, devendo existir ações com divulgação das informações de busca de novas alternativas para conscientização ambiental. 
Nesse contexto, a pesquisa teve como objetivo identificar o manejo e gerenciamento dos resíduos gerados por pacientes diabéticos usuários de insulina análoga do município de Juazeirinho-PB.

\section{MÉTODO}

O trabalho caracterizou-se como um estudo de prevalência, com abordagem quantitativa, e foi realizado no Município de Juazeirinho, localizado na microrregião do Seridó Paraibano, nos meses de junho a dezembro de 2019.

Participaram da pesquisa todos os pacientes diabéticos em uso de insulina cadastrados na Secretaria de Saúde da cidade citada acima, mediante aceitação declarada pela assinatura de Termo de Consentimento Livre e Esclarecido contendo as informações da pesquisa. Para elegibilidade do participante, ele deveria ter mais de 18 anos, ser diabético e estar em uso de insulina com tratamento residencial, não sendo desconsiderados ou discriminados por sexo, posição social, etnia ou religião.

A coleta de dados foi realizada na Farmácia Básica do município após a dispensação da insulina ou análogos, assim como dos materiais para monitoração da glicemia. Foi empregado um formulário de perguntas objetivas e subjetivas para obtenção das informações sobre a insulinoterapia praticada e formas de descarte dos RSS gerados, dados clínicos, sóciodemográficos e hábitos de vida dos voluntários participantes.

O estudo obedeceu a Resolução n 466/2012 do Conselho Nacional de Saúde e só foi desenvolvido após a aprovação pelo Comitê de Ética em Pesquisa do Centro de Ensino Superior e Desenvolvimento (CESED), recebendo a CAAE $n^{\circ}$ 11669519.0.0000.5175. 


\section{RESULTADOS E DISCUSSÃO}

A pesquisa foi realizada com 23 indivíduos diagnosticados com diabetes mellitus e cadastrados na secretaria de saúde e usuários de insulina ou análogos. Observou-se um predomínio do sexo masculino, com idade média de 60 anos e ensino fundamental incompleto, contrapondo-se aos estudos com porcentagem de $52 \%, 67,7 \%$ e $66 \%$ realizados no Rio de Janeiro, São Paulo e Fortaleza, identificando predominância do sexo feminino (SANTOS; SANTOS; PALERMO 2019; SANTOS; RUIZ, 2018; FECHINE et al., 2017). Conhecidos fatores de risco para o diabetes, como o tabagismo e consumo de bebidas alcoólicas foi quase inexistente.

Quanto a faixa etária, observou-se uma idade média de 60 anos, dado que corrobora com Santos e Ruiz (2018) que apresentou um percentual de 58,54\% de idosos, André e Takayanagui (2015), os quais apresentaram uma maior incidência do DM na população acima de 40 anos.

$\mathrm{Na}$ Tabela 1, encontra-se a tipificação dos participantes quanto as informações socioeconômicas, clínicas e de hábitos de vida.

Apenas $32 \%$ dos entrevistados praticam atividades físicas regularmente e evidenciou- se que $92 \%$ dos pacientes diagnosticados com diabetes mellitus fazem dieta com baixo consumo de açúcar. Segundo Paixão, Fernandes e Ferreira (2018), o baixo nível de atividade física e o sedentarismo são fatores de risco tão relevantes quanto uma dieta inadequada, tendo um paralelo direto com o aumento dos diagnósticos de DM em adultos e independe do índice de massa corporal ou do histórico familiar.

Dos 23 pacientes, 60,87\% foram diagnosticados com Diabetes melittus tipo 2. O DM tipo 2 corresponde a maior parcela de diagnósticos de DM no mundo. Este tipo de diabetes afeta indivíduos a partir de 40 anos, cerca de 90 a 95\% dos diagnósticos do DM 2 se dão a partir desta idade, embora possa acometer indivíduos mais jovens. (SBD, 2019). Esta pesquisa revelou que $78,26 \%$ dos pacientes foram diagnosticados com DM há mais de 10 anos assim como no estudo de Fechine et 
al., (2017) com uma prevalência de $87,6 \%$ de diagnósticos há mais de 10 anos. Coelho (2019) apresentou 58\% da população com diagnostico no mesmo intervalo de tempo. Este dado confirma o DM 2 na população tendo em vista a idade média da população, bem como o tempo de diagnóstico.

Tabela 1 - Caracterização socioeconômica, clínica e de hábitos de vida dos 23 participantes.

\begin{tabular}{|c|c|c|}
\hline Variáveis & $\mathbf{n}$ & $\%$ \\
\hline \multicolumn{3}{|l|}{ Sexo } \\
\hline Feminino & 11 & 47,83 \\
\hline Masculino & 12 & 52,17 \\
\hline \multicolumn{3}{|l|}{ Escolaridade } \\
\hline Fundamental incompleto & 16 & 69,56 \\
\hline Fundamental Completo & 1 & 4,35 \\
\hline Médio Incompleto & 1 & 4,35 \\
\hline Médio Completo & 4 & 17,39 \\
\hline Superior Completo & 1 & 4,35 \\
\hline \multicolumn{3}{|l|}{ Fumante } \\
\hline Sim & 1 & 4,35 \\
\hline Não & 22 & 95,65 \\
\hline \multicolumn{3}{|l|}{ Elitismo } \\
\hline Sim & 1 & 4,35 \\
\hline Não & 22 & 95,65 \\
\hline \multicolumn{3}{|c|}{ Prática de atividade física regularmente } \\
\hline Sim & 6 & 26,09 \\
\hline Não & 17 & 73,91 \\
\hline \multicolumn{3}{|c|}{ Dieta com baixo consumo de açúcar } \\
\hline Sim & 21 & 91,3 \\
\hline Não & 2 & 8,7 \\
\hline \multicolumn{3}{|c|}{ Tempo diagnóstico de diabetes mellitus (anos) } \\
\hline 01 a 5 & 3 & 13,04 \\
\hline 5 a 10 & 2 & 8,7 \\
\hline mais de 10 & 18 & 78,26 \\
\hline \multicolumn{3}{|l|}{ Tipo de Diabetes } \\
\hline Tipo 1 & 9 & 39,13 \\
\hline Tipo 2 & 14 & 60,87 \\
\hline
\end{tabular}

Há um alto nível de baixa escolaridade nos usuários de insulina do município, $69,56 \%$ não concluíram o ensino fundamental, fato que pode interferir no tratamento da patologia, bem como na compreensão de orientações. Diversos estudos também 
apresentam uma população com baixo nível de escolaridade. Isto pode ser explicado pela diretriz da Sociedade Brasileira de Diabetes (2019), a qual expõe pesquisas realizadas no ano de 2013 pelo Instituto Brasileiro de Geografia e Estatística e pelo Ministério da saúde, estimou que $6,2 \%$ da população Brasileira, com 18 anos de idade ou mais, referiu diagnostico medico de diabetes, em indivíduos $(9,6 \%)$ sem instrução ou com ensino fundamental incompleto.

Com relação ao acondicionamento e descarte dos resíduos gerados pelo uso da insulina e dos materiais para monitoração glicêmica, estão melhor dispostos na Tabela 2.

Tabela 2. Acondicionamento e descarte dos RSS gerados pela insulinoterapia e monitoração glicêmica pelos participantes do estudo.

\begin{tabular}{lcc} 
Variáveis & $\mathbf{n}$ & $\mathbf{\%}$ \\
\hline Houve orientação sobre o descarte dos resíduos & 5 & 21,60 \\
Sim & 18 & 78,40 \\
Não & 21 & 100,00 \\
Tipo de resíduo & 19 & 47,80 \\
Seringa & & \\
algodão & 9 & 39,10 \\
Armazenamento do resíduo & 14 & 60,90 \\
Saco plástico & & \\
Garrafa PET & 21 & 91,30 \\
Local onde dispõe o resíduo & 2 & 8,70 \\
Lixo comum & & \\
Entrega na Unidade básica de Saúde & 13 & 56,50 \\
Tempo de armazenamento do resíduo & 3 & 13,00 \\
Até o dia da coleta de lixo & 7 & 30,50 \\
Todo dia retira de casa & & \\
Até encher o recipiente & &
\end{tabular}

Fonte: Dados do estudo, 2019.

Verificou-se que a maioria não recebeu orientações sobre o melhor acondicionamento e descarte dos RSS gerados residencialmente, mesmo todos os pacientes em uso de seringas e produzido algodões sujos de sangue. Do total, apenas 13 disseram realizar a monitoração da glicemia e, além das seringas, geraram lancetas descartáveis como resíduos.

Este dado diverge da pesquisa de Santos, Santos e Palermo (2019), onde todos os participantes realizavam monitoramento do nível glicêmico nas residências. 
Ainda sobre a importância do auto monitoramento os autores ressaltaram a importância do controle residencial para o DM tipo 1 e tipo 2 e a frequência das verificações deve ser adaptada de acordo com o perfil de cada paciente. $O$ controle metabólico adequado é um desafio para os profissionais que acompanham os diabéticos, pois deve ser considerado o fato dos cuidados diários serem realizados por pacientes idosos ou familiares necessitando de acompanhamento continuo para promoção do autocuidado (FECHINE, et al., 2017).

Sobre o armazenamento dos resíduos gerados, mais da metade afirmou armazenar os perfurocortantes em garrafas PET e uma boa parcela apenas descartavam em sacos plásticos de lixo comuns. Situação agravada, pois quase cem por cento encaminhava os RSS para coleta municipal de lixo.

Em relação as ações de educação em saúde, no que se refere a Resíduos de Serviços de Saúde - RSS, 78,4 \% afirmaram nunca ter recebido orientações sobre o descarte dos resíduos gerados pela aplicação de insulina e do monitoramento dos níveis glicêmicos realizados em suas residências. As ações de educação em saúde merecem destaque para pacientes diagnosticados com DM, visto que estes necessitam de instruções que fundamentam seu tratamento para realizarem corretamente a aplicação da insulina análoga e o controle adequado da glicemia (ANDRE; TAKAYANAGUI, 2015). No estudo de Santos, Santos e Palermo (2019) houveram falhas de comunicação entre profissionais de saúde e usuários, consequentemente ausência de informação e orientação sobre a condição crônica do paciente.

Dos resíduos provenientes da aplicação da insulina análoga e do monitoramento da glicemia capilar gerados pela população estudada, $91,3 \%$ descartavam no lixo comum seringas lancetas e algodão corroborando com estudos realizados no Rio de Janeiro, São Paulo, Minas Gerais, (SANTOS; SANTOS; PALERMO, 2019; AQUINO; ZAJAC; KNIESS, 2019; FRANCO et al., 2016).

No estudo, constatou-se que $60,90 \%$ armazenam os resíduos vasilhas plásticas do tipo garrafas PET e 91,3\% jogavam esses recipientes no lixo comum, sendo que $(56,5 \%)$ deste retiravam as embalagens todas as vezes que a coleta do lixo passava. É recomendado o descarte de agulhas e lancetas em recipiente próprio para material perfurocortante com paredes rígidas resistentes a perfuração, boca 
larga e tampa, fornecidos pela Unidade Básica de Saúde - UBS ou recipiente rígido resistente (BRASIL 2013). Entretanto a Sociedade Brasileira de Diabetes não recomenta uso de garrafas PET para descarte, frisando a inadequação para o descarte de resíduos E (SDB, 2019).

A UBS é a principal porta de entrada, é o centro de comunicação da população, nesse contexto os gestores devem apoiar e implantar programas de educação em saúde nos serviços destinados a pacientes diabéticos e ainda fornecer recipientes adequados para acondicionamento dos resíduos (AQUINO; ZAJAC; KNIESS, 2019).

$\mathrm{Na}$ Tabela 3, verifica-se o tratamento realizado pelos pacientes, assim como a frequência de administração de insulina e realização da monitoração glicêmica, o que indica o volume de RSS gerados pelos participantes do estudo.

Tabela 3. Linhas de tratamento dos pacientes e frequência de monitoração glicêmica.

\begin{tabular}{|c|c|c|}
\hline Características da pratica de insulinoterapia & $\mathrm{n}$ & $\%$ \\
\hline \multicolumn{3}{|l|}{ Controle medicamentoso } \\
\hline Insulina & 8 & 34,80 \\
\hline Insulina + metformina & 10 & 43,50 \\
\hline Insulina + outro medicamento & 5 & 21,70 \\
\hline \multicolumn{3}{|l|}{ Tipo de insulina } \\
\hline Neutral Protamine Hagedorn (NPH) & 19 & 82,60 \\
\hline Associação da regular + NPH & 4 & 17,40 \\
\hline \multicolumn{3}{|l|}{ Frequência de administração (ao dia) } \\
\hline 1 vez ao dia & 5 & 21,74 \\
\hline 2 vezes ao dia & 17 & 73,91 \\
\hline 3 vezes ao dia & 1 & 4,35 \\
\hline \multicolumn{3}{|l|}{ Frequência com que verifica a glicemia capilar } \\
\hline 01 vez ao dia & 7 & 30,50 \\
\hline 01 vez na semana & 6 & 26,00 \\
\hline Não realiza controle & 10 & 43,50 \\
\hline \multicolumn{3}{|l|}{ Reutilização da seringa descartável } \\
\hline Sim & 16 & 69,60 \\
\hline Não & 7 & 30,40 \\
\hline
\end{tabular}

Fonte: Dados do estudo, 2019.

Para o tratamento do diabetes $43,5 \%$ dos entrevistados fazem o uso de insulina e metformina e $82,6 \%$ usam a insulina do tipo NPH esses dados diferem de 
Fechine et al. (2017) onde, entre seus entrevistados, 60\% utilizavam a associação da insulina regular e da NPH. Foi comum a frequência de administração da insulina duas vezes ao dia. Conforme a Sociedade Brasileira de Diabetes (2019) a combinação de insulinoterapia com antidiabéticos orais tem como potencial vantagem a melhor aceitação da insulina pelo paciente, uma vez que evita múltiplas injeções diárias.

Coelho (2019) explicou em sua pesquisa que o tratamento farmacológico do DM é dividido em antidiabéticos orais que ajudam no controle glicêmico e as insulinas de ação rápida (regular) e de ação intermediária (NPH - Neutral Protamine Hagedorn), ambas são disponibilizadas pela atenção básica. Logo, o fato de ser disponilizada pelo Serviço Único de Saúde faz com que seja mais usada que as demais. A metformina, antidiabético oral, faz parte do plano terapêutico de $43,5 \%$ dos usuários, isto porque é um medicamento usado como primeira opção terapêutica, tem perfil de segurança e capacidade de reduzir eventos macro vasculares (BRASIL, 2013).

Ao serem questionados sobre o reaproveitamento da agulha, mais da metade afirmou reaproveitá-las. O fato da maioria dos diabéticos reaproveitarem o material usado para aplicação de insulina corrobora com as pesquisas que mostram a reutilização como algo comum, há casos que a reutilização se dá até três vezes (FECHINE et al. 2017; SANTOS, SANTOS E PALERMO, 2019;).

O Ministério da Saúde elucida que mesmo seringas e agulhas sendo descartáveis podem ser reutilizadas pela mesma pessoa desde que não haja contaminação nos objetos. A quantidade de vezes que a agulha pode ser reutilizada pode variar de acordo com o fabricante, porem deve ser trocada ao causar desconforto ao paciente e após o uso a seringa deve ser recapada, recomenda-se que a agulha não seja higienizada com álcool (BRASIL 2013) Entretanto, Frid et al., (2016) citado por Santos, Santos e Palermo (2019) afirma que pesquisas mostram que a administração por vias parenterais não devem reutilizar os materiais para aplicação, uma vez que o paciente ficará mais suscetível a infecção e dor durante e aplicação. 


\section{CONCLUSÃO}

Ao analisar a população estudada, foi observado que a falta de orientação ao usuário é um dos principais problemas para o descarte inadequado dos RSS. Notase também que a população tem um baixo nível de escolaridade, necessitando de uma maior atenção dos profissionais de saúde para as orientações necessárias ao autocuidado com pacientes diagnosticados com diabetes mellitus.

A falta de legislação para resíduos de saúde produzidos em residências, também é um agravo, uma vez que não obriga nenhum estabelecimento de saúde a receber os materiais provenientes de pacientes com complicações crônicas como o DM.

Faz-se necessária a conclusão da pesquisa afim de concluir a prevalência das variáveis sociodemográficas e clinicas da população cadastrada na secretaria de saúde do município de Juazeirinho-PB. Ademais, é de extrema importância o investimento na educação em saúde para todos os profissionais, uma vez que estes passarão as informações para a população de acordo com a necessidade de entendimento de cada um. 


\section{REFERÊNCIAS BIBLIOGRÁFICAS}

ANDRÉ, S. C. S.; TAKAYANAGUI, A. M. M. Orientações sobre o descarte de resíduos gerados em domicílios de usuários de insulina. Revista Baiana de Saúde Pública, v.39, n.1, p.105-118, 2015.

AQUINO, S; ZAJAC, M. A. L.; KNIESS, C. T. Percepção de diabéticos e papel dos profissionais de saúde sobre a educação ambiental de resíduos perfurocortantes produzidos em domicílios. Revista Brasileira de Educação Ambiental, v.14, n.1, p.186-206, 2019.

BRASIL. Ministério da Saúde. Agencia Nacional de Vigilância Sanitária. RDC n 222 de 28 de março de 2018. Brasília: ANVISA, 2018.

BRASIL. Ministério da Saúde. Secretaria de Atenção a Saúde. Estratégias para o cuidado da pessoa com doença crônica: diabetes mellitus. Brasília, 2013.

COELHO, F. M. Educação em saúde no manejo de resíduos sólidos gerados por usuários de insulinoterapia. Departamento de pós-graduação em ensino de stricto sensu. Mestrado em Ambiente e Saúde, P.84 Universidade de Cuiabá - Unic, 2019.

FECHINE et al. Resíduos de insulinoterapia produzidos no domicílio de diabéticos acompanhados na Atenção Primária. Revista Brasileira de Enfermagem, v.70. n.3, p.646653, 2016.

FRANCO et al., Avaliação do descarte de perfurocortantes por pacientes usuários de insulina atendidos por uma drogaria de Viçosa- MG. Revista Cientifica Univiçosa, v.8, n.1, p.192- 197, 2016.

PAIXÃO, A. F.; FERNANDES, L. A.; FERREIRA, L. Nível de atividade física, percepção da qualidade de vida e sono em diabéticos mellitus II no município de bebedouro/SP. Centro Universitário Unifafibe. São Paulo, 2018.

SANTOS, L. N. dos; RUIZ, J. B. Gerenciamento dos resíduos de saúde produzidos por diabéticos insulinodependentes do município de Umuarama - PR. . v.13, n.2, 2018.

SANTOS, T. N.; SANTOS, C. M.; PALERMO, T. A. C. Resíduos de saúde nos domicílios de pacientes com diabetes mellitus. Revista Laborativa. v.8, n. 1, p. 44-56, 2019.

SOCIEDADE BRASILEIRA DE DIABETES. Diretrizes da sociedade Brasileira de Diabetes 2019-2020. São Paulo: SBD, 2019. 